\title{
MANAGEMENT OF MICROGRIDS IN MARKET ENVIRONMENT
}

\author{
A.G. Tsikalakis ,A.Dimeas, N. D. Hatziargyriou, J.A. Pecas Lopes, G.Kariniotakis , \\ J.Oyarzabal
}

\author{
SCHOOL OF ELECTRICAL \& COMPUTER ENGINEERING \\ NATIONAL TECHNICAL UNIVERSITY OF ATHENS9, \\ 9 Iroon Polytechneiou Str., GR-15773 Zografou, Athens, GREECE \\ Phone.: (30) 2107723696, Fax: (30) 2107723968 \\ E-Mail: atsikal@power.ece.ntua.gr
}

Keywords: Demand side bidding, Distributed Generation, Forecasting, Markets, Microgrids, Security

\begin{abstract}
Restructuring of power markets has helped in the penetration of Distributed Generation (DG) in the electricity networks. Microgrids are Low Voltage distribution networks comprising various distributed generators (DG), storage devices and controllable loads that can operate interconnected or isolated from the main distribution grid, as a controlled entity. This paper describes the main functions of the Microgrid Central Controller required for the optimization of Microgrid operation during interconnected operation. This is achieved by maximizing its value, i.e. optimizing production of the local DGs and the exchange of power with the main distribution grid.
\end{abstract}




\section{INTRODUCTION}

The deregulated energy environment, among other effects, has favored a gradual transition from centralized power generation to Distributed Generation (DG) sources connected at the Medium Voltage or Low Voltage side of the Distribution Network [1]. These DG sources comprise several technologies, such as Diesel Engines, Micro Turbines and Fuel Cells either in CHP operation or purely for electricity production, Photovoltaics, small Wind Turbines, etc. The capacity of the DG sources typically varies between few kWs and 1-2 MWs.

The coordinated operation and control of DG sources together with controllable loads and storage devices, such as flywheels, energy capacitors and batteries is central to the concept of Microgrids. Microgrids can operate interconnected to the main Distribution grid, or in an autonomous way, if disconnected from the main grid, in case of external faults. From the grid's point of view, a Microgrid can be regarded as a controlled entity within the power system that can be operated as a single aggregated load [2,3] and, given attractive remuneration, as a small source of power or ancillary services supporting the network. From a customer point of view, Microgrids similar to traditional LV distribution networks provide their thermal and electricity needs, but in addition, enhance local reliability, improve power quality by supporting voltage and potentially lower costs of energy supply and, overall emissions of the power system they are connected to, if DG sources are mainly based on RES.

This paper describes the functions required for the operation of the Microgrids Central Controller (MGCC) in the interconnected mode, in particular:

- Economic Scheduling

- Forecasting (short term) for electrical load, RES power production and heat

- Security Assessment

- Demand Side Management (DSM) Functions

The functions of the MGCC in islanding mode of operation, like islanded operation control, synchronizing of the Microgrid system with the main, black start capabilities, are not dealt with in this study.

Also, a flexible software tool for the optimization of the Microgrid operation has been developed and typical results are shown at the last sections of the paper. 


\section{TYPICAL MICROGRIDS CONTROL STRATEGIES}

A hierarchical control architecture comprising three critical levels as shown in Figure 2.1, is assumed. It comprises :

- Local Controllers (LC) either Micro Source Controllers or Load Controllers.

- MicroGrid System Central Controller (MGCC)

- Distribution Management System (DMS).

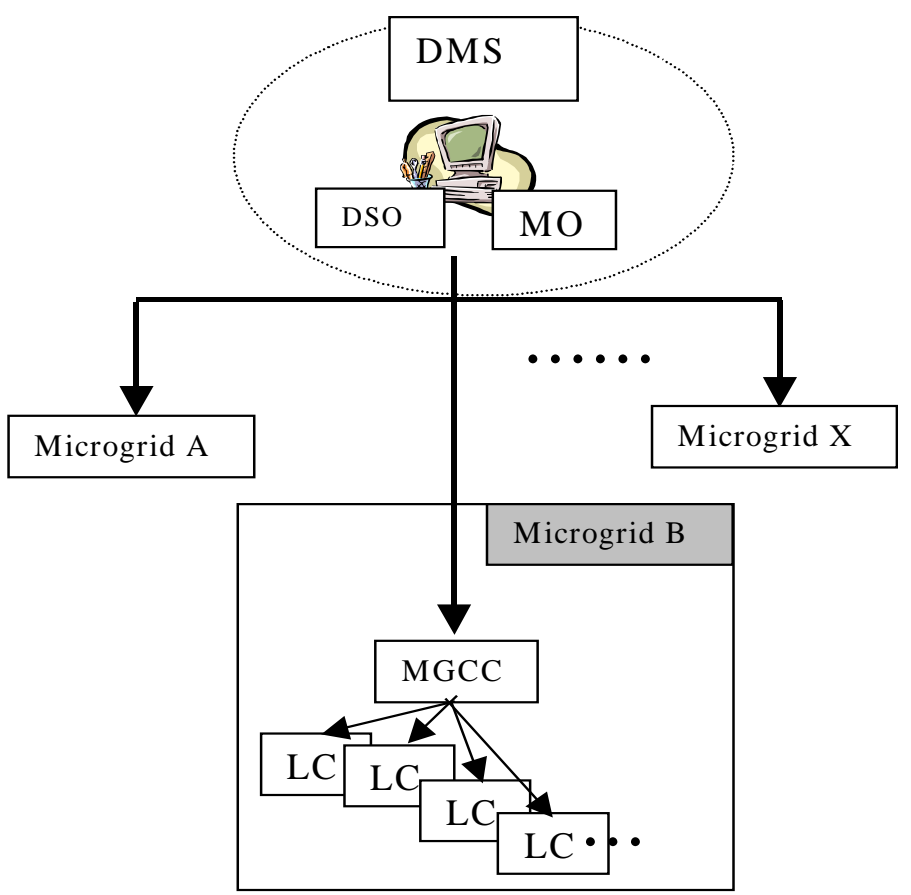

Figure 2.1: Microgrid control architecture

Depending on the distribution of functional responsibilities between the LCs and the MGCC, the Microgrid operation can be perceived ranging from a fully decentralized to a centralized approach. Both approaches have their pros and cons, as discussed in [4].

The Micro Source Controllers use local information to control the voltage and the frequency of the microgrid in transient conditions. In a centralized control approach, they follow the demands from the MGCC, when connected to the power grid, and have the autonomy to perform local optimization of the micro source active and reactive power production, and fast load tracking following an islanding situation. Local Micro Load Controllers are installed at the controllable loads to provide load control capabilities following demands from the MGCC, under a Demand Side Management (DSM) policy or for load shedding. 
In a fully decentralized approach, LCs assume the main responsibility to maximize their production in order to satisfy the demand and probably provide the maximum possible export to the grid taking into account current market prices. This approach is based on decentralized multi-agent technology and its realization is explicitly described in [5].

The Microgrid Central Controller (MGCC) is responsible for the maximization of the Microgrid value and the optimization of its operation. If centralized control is adopted, it uses the market prices of electricity and probably DSM requests to determine the amount of power that the Microgrid should draw from the distribution system, optimizing the local production capabilities. It might use simple load forecasts (electric and possibly heat) and forecasts of power production capabilities. The defined optimized operating scenario is achieved by controlling the micro-sources and controllable loads in the Microgrid by sending control signals to the field. In this framework, non-critical, controllable loads can be shed, when necessary. Furthermore, it is necessary to monitor the actual active and reactive power of the components. These techniques can be considered equivalent to the secondary control of the interconnected grid.

In a decentralized control approach there is only need for an extra supervisory Agent whose primary job is to record the bids of the Micro sources and the power flow. A transaction is valid only if it is registered in the supervisory agent and this is vital in order to avoid double bids to individual loads. In Market Operation its job is to create the final bill for each load or unit.

The DMS consists of the Distribution System Operator (DSO) and one or more Market Operators (MO), who can be the same or a different entity. The DSO is responsible for the operation of medium and low voltage areas in which more than one Microgrid may exist. The MO is responsible for the market functions, like determining the external clearing market prices for the specific area operated by the DSO.

\section{MGCC OVERALL ORGANIZATION}

The following diagram describes a possible operation of the MGCC under a centralized control approach. It is assumed that the MGCC acts as a market operator for the controlled area of the Microgrid. The local controller MC takes into account the operational cost function of the micro-source and the prices of the market provided by the MGCC, in order to make offers to MGCC and provide the limits of production. These offers are made at 15 minutes interval for the next few hours, i.e. the optimization horizon.

The MGCC takes into account:

- The external market prices (actual-e.g day-ahead market or forecasted)-or the tariff scheme outside the microgrid. 
- The bids of the micro-sources.

- The suggested limits of production

- The demand side bidding for "low" and "high’ priority loads

and solves the optimization problem, as defined in Section 4.

The MGCC, after the optimization process is complete, sends to the Local Controllers:

- The external market prices for the optimization horizon at 15-minutes steps

- The set-points for active and reactive power.

- The load to be shed or served according to Demand side bidding option followed.

The information flow between the MGCC and the MCs is shown in Figure 3.1.

The energy market prices may come from the data of a day-ahead market or be the result of price forecasting functions.

It is assumed that some sort of contract presumably binds the owners of the MGCC and the energy producers/users, so that set-points and load shedding are implemented by releasing control of the generation and loads to be shed to the MGCC.

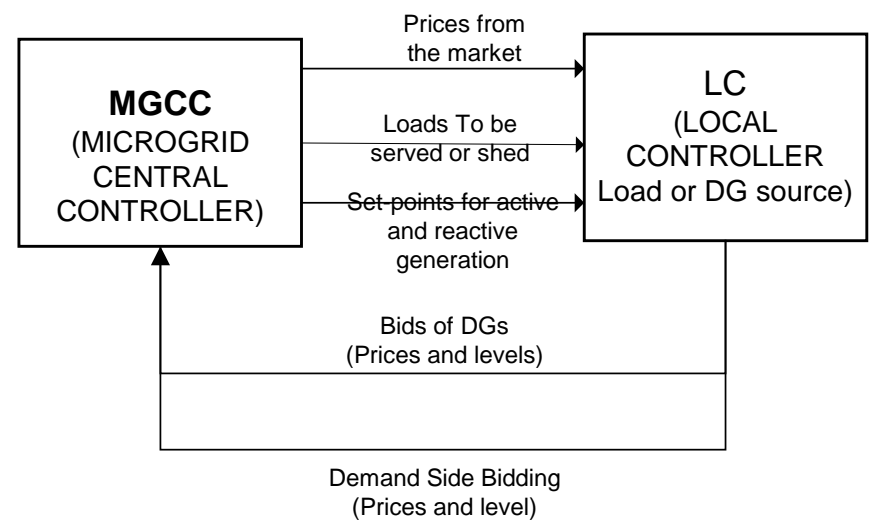

Figure 3.1: Information flow between the MGCC and the LCs

\section{MARKET ASPECTS}

Two Market policies are assumed in our study. In the first policy, the MGCC aims to satisfy the local energy demand using its local production, when financially beneficial, without exporting power to the upstream distribution grid [3] trying to reduce the energy cost of the whole Microgrid. For the overall distribution system operation, such behavior is beneficial, because at the time of peak demand leading to high electricity prices, the Microgrid relieves possible network congestion by 
partly or fully supplying its energy needs. From the end-user point of view, the MGCC minimizes operational cost of the Microgrid, taking into account market prices, demand and DG bids. The owners of DG sources make profit from the difference between the bid price they suggest and the operating cost of their unit. The customers of the Microgrid share the benefits of reduced operational costs since they buy active power at lower prices compared to no Microgrid operation.

In this case, MGCC is provided with:

- The external market prices (A €ct/kWh)

- The power demand, probably as a result of a short term load forecasting tool.

- The bids of the microsources.

The MGCC tries to minimize the energy costs for the whole Microgrid for each of 15 minutes interval subject to constraints such as active and reactive power balance, P-Q curve for each one of the generator units and the grid, technical limits of each unit, etc.

In the second policy, the Microgrid participates in the energy market of the distribution area, buying and selling active and reactive power to the grid, probably via an Aggregator or similar Energy Service provider. According to this policy the MGCC tries to maximize the corresponding revenues of the Aggregator, by exchanging power with the grid. The customers are charged for their active and reactive power consumption at market prices. The Microgrid behaves as a single generator capable of relieving possible network congestion, not only in the Microgrid itself, but also by exporting energy to nearby feeders of the distribution network. The owners of the DG sources make profit due to difference of the bid price and the operating cost of their own unit. The revenues of the Aggregator are, either the profit of the energy provider or, if the Aggregator is a co-operation of the end-users of the Microgrid, are distributed to those having shares of this cooperative structure.

The MGCC is provided with:

- The market price for buying and selling energy to the grid. The same prices apply to the consumers within the Microgrid.

- The power demand, probably from a short-term forecasting tool

- The bids of each microsource regarding active power.

- The maximum capacity allowed to be exchanged with the grid. This can be for example some contractual agreement of the Aggregator or the physical limit of the interconnection line to the grid.

In this case, the objective function expresses the Maximization of the Microgrids value, expressed as the difference between Income and Expenses, subject to similar constraints, as in the previous policy plus constraints related to the capacity of the 
interconnection and the active power possibly contracted by the Aggregator. In both policies, demand side bids and adequacy or power quality issues can be included in the optimization routines.

\section{DEMAND SIDE BIDDING}

It is assumed that at least some of the customer loads are equipped with load controllers. Each consumer may have low and high priority loads and sends separate bids to the MGCC for each of them. In this way, the total consumption of the consumer is known in advance. Some of the loads will be served and others not, according to the bids of both the consumers and the micro-source producers. For the loads that the MGCC decides not to serve, a signal is sent to the load controllers in order to interrupt the power supply.

Two options are considered for the consumers' bids:

A) Consumers bid for supply of high and low priority loads

B) Consumers offer to shed low priority loads at fixed prices in the next operating periods.

In both options the MGCC:

1. Accepts bids from the consumers every hour corresponding to quarter of an hour intervals.

2. Informs each consumer about acceptance of his bids

3. Informs consumers about the prices of the external market. These prices help preparing the bids. For Microgrid operation as a good citizen, the market prices will be the highest prices, if security constraints are not considered.

In both options, if any load bids at prices higher than both the external market and the generators bidding prices, this load will be served paying at the Microgrid market clearing price as defined by the market policy followed within the Microgrid.

\subsection{Option A}

It is assumed that each consumer places bids for his own load in two levels and the prices of the bids reflect his interest for each load block. The "low" priority loads are the ones the consumer prefers not to operate when the market prices are high, and can be served later, when prices are lower (shift) or not served at all (curtailment). The MGCC knows the total volume of low and high priority loads and their price level and then decides which of them to serve and which not, based on the optimization function outcome. The MGCC aggregates the demand bids, the production bids and the external market prices and decides which bids will be accepted. The total demand of the Microgrid is the summation of the accepted demand bids making short-term load forecasting is less relevant. Information about 
the external market prices influences consumer bids, i.e. might shift load for a while in order to achieve lower costs for his electricity consumption.

\subsection{Option B}

In this case each consumer states the amount of load that can be shed in the next operating period. It is assumed that load can be shed in maximum two steps. The consumer will be compensated for his service, if his bid is accepted. In this option the MGCC has the right to shed "cheaper" loads, if they are on. Loads to be shed are considered as "negative" generation, if they are cheaper than actual generation, lowering the total demand. The MGCC takes into account the bids for shedding, the bids of production and either the aggregation of the total demand as the actual total demand to be met or a forecasted demand and accepts the demand shedding bids. The total demand finally to be met in the Microgrid is the actual demand minus the accepted demand shedding bids.

\section{SOLUTION METHODS}

In order to optimize the operation of the microgrid two functions are required:

- Unit Commitment (UC) function, that determines which micro-sources will be committed at each time interval

- Economic Dispatch (ED) function, that decides the operating point (power) of each production unit (and load, if applicable).

The reason for applying both UC and ED is the fact that some units may have low variable cost, but high constant cost, For example, a unit may be expensive to start up, but once committed it might have lower variable cost than the rest committed units. Thus, it is more economical to operate at higher loading compared to the rest. In this case, an ED function is required to further optimize the economic operation of the Microgrid.

In the Microgrid studies the following techniques have been used to solve the above problems.

- Priority list

- Sequential Quadratic Programming (SQP).

- Ant-colony optimization

In the following, the basic steps of the UC and ED functions are described:

\subsection{UC function}

1. Reads minimum and maximum capacity of the production units

2. Reads external market prices. The grid (external market) is considered as a

"virtual", large generator with maximum capacity determined by the congestion 
limit of the interconnection. Therefore the units taking part in the market are number of micro-sources +1 .

3. Creates priority list sorted according to the differential cost of each unit - ratio of cost in maximum capacity to maximum capacity of each one of the microsources.

4. The cheapest units are committed until the demand plus an amount of spinning reserve are covered. One of these units is the grid.

\subsection{ED function}

1. Reads minimum and maximum capacity of the production units

2. Reads external market prices. As above, the external market is considered as a "virtual" generator with maximum capacity the congestion limit of the interconnection.

3. All committed units are dispatched at least at their technical minima.

4. The rest of the active demand, if the summation of the technical minima of the committed units is subtracted from the actual demand, is dispatched to the committed units, according to their bid prices, so that the active power demand is met.

\section{FORECASTING FUNCTIONS}

The operation of a Microgrid via an MGCC needs inputs about the electricity and heat demand, the possible RES production and the electricity prices. Depending on the mode of operation of a Microgrid it is clear that in isolated mode prediction of demand is of primary importance, since the aim is to achieve the balance of the system. In a non-isolated mode the importance of predicting the demand or the generation may change if one considers a system-driven or a customer-driven approach. In the first case, forecasting functions may have less importance since one may consider that a Microgrid after all is connected to an "infinite" source of power able to cover any deficit at any moment. In a customer-driven approach however, economics and thus forecasting gain in importance. Then, if the Microgrids is the "business-case" i.e. of an energy service provider who has to consider electricity prices, then a tool for taking decisions based on forecasting will be needed. Forecasting functions are expected to gain in importance when one considers multi-Microgrids scenarios. In any case, in the scale of a microgrid, one should consider cost effective approaches for forecasting. Today forecasting technology is an expensive one and forecasting tools are not plug \& play ones.

Forecasting in Microgrids is mainly short-term with high temporal resolution (i.e. 10 minutes) and for the next 1-4 hours. However, unlike large interconnected systems or islands [6,7], the aggregation or smoothing effect is reduced increasing uncertainty as the size of the Microgrid gets smaller. In this paper persistence 
method is applied. This is the simplest method assuming that the predicted variable will remain the same as during the previous period:

$$
\hat{P}(t+k)=P(t), \mathrm{k}=1,2, \ldots .
$$

A more sophisticated approach based on adaptive fuzzy neural networks has been also developed giving the flexibility to consider various input variables according to the availability of data.

Similar methods can be used for wind or other RES forecasting although methods using meteorological data have been applied for longer term prediction and much larger power systems than a Microgrid [7-9]. One of the main motivations for developing distributed generation and especially in the microgrids option is to achieve higher energy efficiency by combining heat and power demand. Thus, forecasting heat consumption is a necessary functionality that has to be provided to the MGCC for optimizing decisions on covering this demand. The heat demand forecasting developed takes into account the following parameters [10]:

- time of day effect,

- weekend/weekday effect

- seasonal effects

- time varying volatility

- high negative correlation between heat demand and outside temperature.

The simpler approaches developed so far for on-line prediction of heat consumption in district heating systems are based on purely ARIMA models that use only heat demand data as input. SARIMA models considering seasonal differencing has been also applied. As an extension ARIMAX models are also considered with temperature as an explanatory variable.

Another concern is the electricity price forecast, since the bids of the DG sources are submitted accordingly. This is required if no information about the external market prices is available or if the Microgrid participates in the clearing market. Electricity prices as studied by several Exchange markets, share the same characteristics that help in their forecasts [11].

- strong mean reversion: deviation of the price due to random effects are corrected to a certain degree.

- time of the day effect,

- calendar effects such as weekdays, weekends and holidays,

- seasonal effects,

- time varying volatility and volatility clustering

- high percentage of unusual prices mainly in periods of high demand, 
- inverse leverage effect: a positive price shock has a bigger impact than a negative one.

- non-constant mean and variance

Some of the models that have been applied for short-term price forecasting include:

- mean reverting processes

- mean reverting processes with time-varying mean

- autoregressive moving average models (ARMA)

- exponential generalized autoregressive conditional heteroscedasticity models (EGARCH)

\section{ON -LINE SECURITY ASSESSMENT FUNCTIONS}

On-line security assessment provides a way to evaluate the robustness of the LV Microgrid to survive when a sudden disconnection from the MV network occurs. Such a sudden change on the system's operating conditions must be quickly and efficiently compensated by both microsources and loads in order to avoid unacceptable frequency and voltage variations. It should be noted that in power electronics dominated Microgrids, the limits of these variations might be possibly more relaxed compared to classical power systems, nevertheless limits do exist, in order to maintain acceptable quality of service and to avoid possible loss of supply.

The generation of functional knowledge (learning set generation) requires the analysis of the dynamic behaviour of the microsources operating together in the Low Voltage (LV) network under a large number of several different operating conditions, during the sudden disconnection from the upstream Medium Voltage (MV) network.

Taking into account the dynamic characteristics of the DG sources and storage devices, the dynamic security of a Microgrid is studied using rules based on Neural Networks (NN) and Decision Trees (DT) two techniques that have been used in similar applications [12-16]. In the following, the Neural Network structure and performance are described.

\subsection{Neural Networks (NN)}

A feed-forward NN architecture was adopted with the following characteristics:

- An input layer with 9 neurons and a hyperbolic tangent activation function

- A hidden layer with 10 neurons and a hyperbolic tangent activation function

- An output layer with 1 neuron and a linear activation function

The training approach was based on a back-propagation approach. 
The best performance was obtained using the following variables as inputs of the $\mathrm{NN}$ :

- Diesel group active power;

- Split-shaft microturbine active power;

- Single-shaft microturbine active power;

- Imported (or exported) active power;

- Active load in the microgrid;

- SOFC reactive power;

- Imported (or exported) reactive power;

- Reactive load in the microgrid;

- Node voltage where the Single-shaft microturbine is connected;

An evaluation of the quality of the NN was performed afterwards using the test set and Table 8.1 summarizes the results from the evaluation of the NN structure for evaluating the robustness of the dynamic security assessment model.

Figure 8.1 shows the data fitness quality in the NN (the real frequency deviations target values - are compared regarding the emulated ones).

Table 8.1 Results from the Neural networks simulation

\begin{tabular}{cc}
\hline Mean Square Error & 0.000125 \\
Mean Absolute Error & 0.0065 \\
\hline
\end{tabular}

The results obtained indicate that the selected variables and the architecture used are sufficient to emulate the index used to characterize the robustness of the Microgrid operation. The architecture used proved to be adequate to accomplish the proposed objective. 


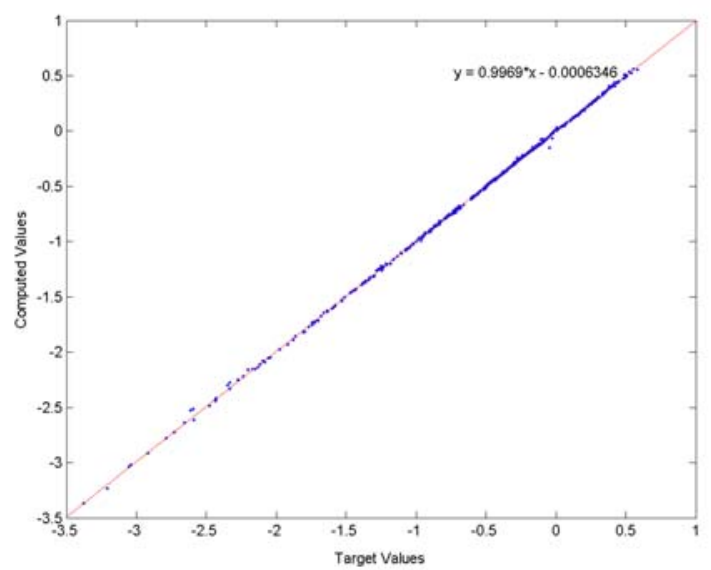

Figure 8.1: NN Performance

\section{STUDY CASE RESULTS}

The above functions have been incorporated in a software package equipped with a friendly Human Machine Interface (HMI). In this Section indicative results obtained from the application of the above methods to the study case LV Microgrid [17] are shown. The network comprises three feeders, one serving a primarily residential area with a variety of DG sources, one industrial feeder serving a small work-shop, and one commercial feeder. The total daily energy demand is $3188 \mathrm{kWh}$. The energy market variations are represented by actual energy prices from the Amsterdam Power Exchange (ApX) [18] for a day with rather volatile prices. In Figure 9.1 the assumed total Microgrid demand, on the left, and the demand per feeder, on the right, are displayed.

In figure 9.2 the results of optimal operation are shown. More specifically, the production of each generator during the optimization step and the grid inflow are displayed and the end-user can click on them and see the exact production of each unit. The user can also display the periods when it is economically beneficial to operate the local microsources and display information about costs and energy balance. 


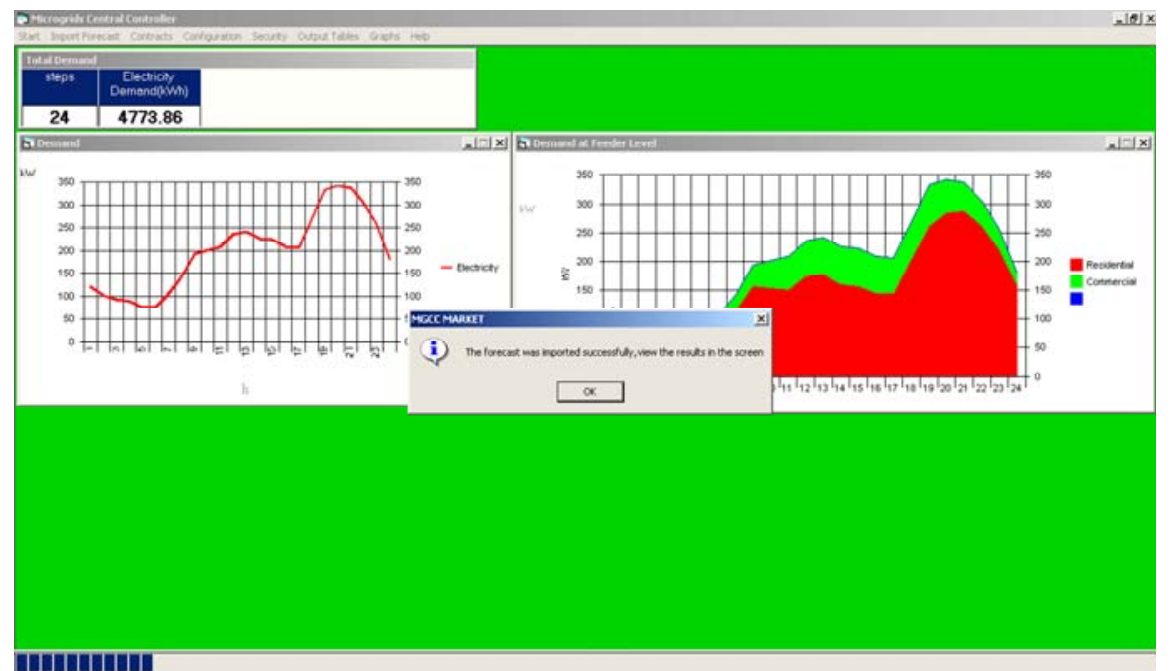

Figure 9.1: The screen for demand

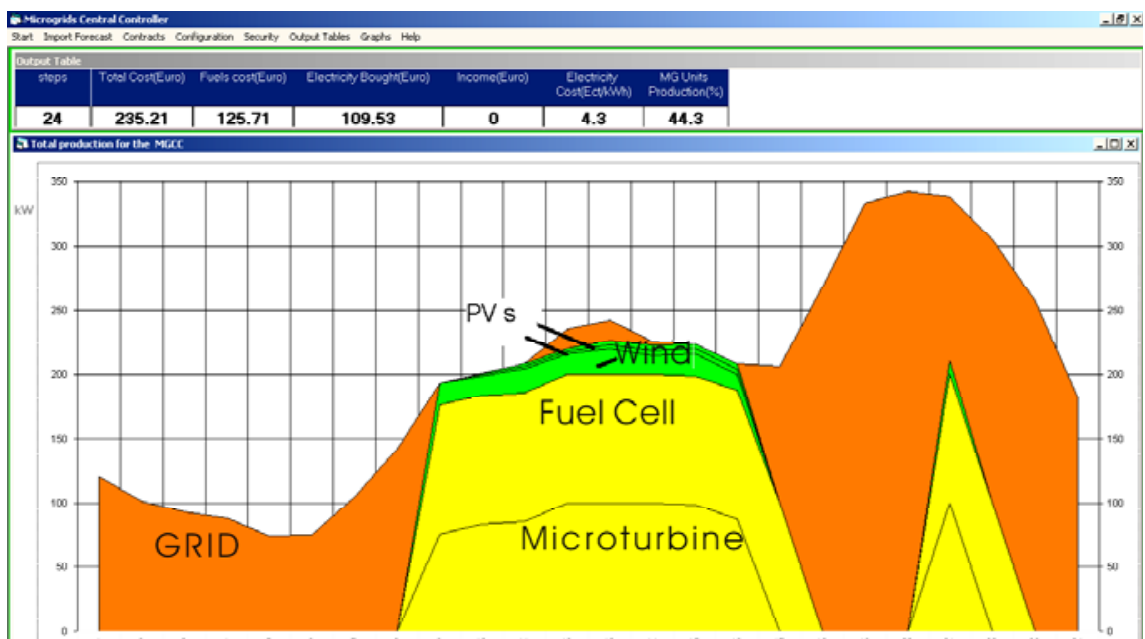

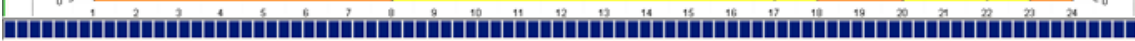

Figure 9.2: The results of optimal operation

As a study case operation of the Microgrid the following cases are examined:

1. There are no micro-sources and all the demand is met by grid imports. Calculated cost 60.13 Euro

2. Good Citizen Policy: Calculated cost $\mathbf{5 2 . 7 4}$ Euro or $12.29 \%$ cost reduction

3. Ideal Citizen Policy: Calculated cost $\mathbf{4 8 . 9 1}$ Euro or $18.66 \%$ cost reduction 
As shown by the negative values in Figure 9.3 energy is sold to the grid between the $11^{\text {th }}$ and the $16^{\text {th }}$ hour.

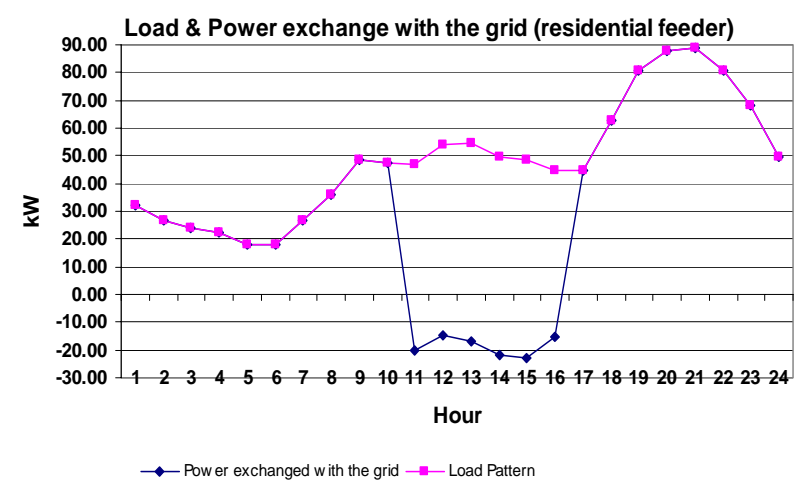

Figure 9.3 : Power Exchange with the grid by the residential feeder

\section{CONCLUSIONS}

This paper describes the main functions required by the Microgrid Central Controller for the efficient Microgrid participation in future real-time markets following different policies. The external market prices and bids coming both from DG sources and loads are considered. The economic scheduling, forecasting and security functions developed within the EU project "Microgrids" are described. The functions developed allow coordinated management of the Microgrid microsources, in order to achieve decrease in their operating costs and making Microgrids a viable future power system prospect. Further research on the business models and the contracts within the participants of the Microgrids will be treated in following paper as a series of dealing with different aspects of Microgrids.

\section{ACKNOWLEDGEMENTS}

The authors express their gratitude to the EC for funding the R\&D project "Microgrids" (Contract No ENK 5-CT-2002-00610).

\section{REFERENCES}

[1] G. Pepermans, J.Driesen, D. Haeseldonckx, R. Belmaans, W. D’haeseleer, "Distributed generation: definition,benefits and issues" Int Journal Energy Policy, Volume 33, Issue 6, April 2005, pp 787-798

[2] A. Tsikalakis, N. Hatziargyriou , Economic Scheduling Functions of a Microgrid Participating In Energy Markets”, Proc of the DG Cigre symposium, Athens,13-16 April 2005,paper no303 
[3] R. Lasseter, A. Akhil, C. Marnay, J. Stephens, J. Dagle, R. Guttromson, A. Meliopoulos, R. Yinger and J. Eto, "White Paper on Integration of Distributed Energy Resources. The CERTS MicroGrid Concept." Consortium for Electric Reliability Technology Solutions (CERTS), CA, Tech. Rep. LBNL-50829, Apr. 2002.

[4] N Hatziargyriou, A.Dimeas, A.Tsikalakis "Hierarchical and distributed control strategies for Micro-grids", Int J. of Distributed Energy Sources,Vol1 , No3 ,2005

[5] A.L.Dimeas, N.D.Hatziargyriou,"Operation of a Multiagent System for Microgrid Control", IEEE Trans. On Power Systems,Vol20,Aug2005,pp14471455

[6] Liu, K. et al, "Comparison of very short-term load forecasting techniques," IEEE Trans. Power Systems, vol. 11, no. 2, pp. 877-882, May 1996.

[7] Kariniotakis G., et al "Load, Wind and Hydro Power Forecasting Functions of the More-Care EMS System", CD Proceedings of the IEEE/IEE MedPower2002 Conference, Athens, Greece, 4-6 November 2002.

[8] Giebel, G., Kariniotakis G., Brownsword, R., "The State-of-the-art in shortterm prediction of wind power. A literature overview", Deliverable Report D1.1 of the Anemos project (ENK5-CT-2002-00665), available online at http://anemos.cma.fr.

[9] X. Wang, G. Sideratos, N. Hatziargyriou, L. Tsoukalas "Wind Power Forecasting for Operational Planning”,CD Proceedings of the $8^{\text {th }}$ PMAPS Confrenece, Iowa,September 2004

[10] Canu, S., Duran, M., and Ding, X., "District Heating Forecast using Artificial Neural Networks", International Journal of Engineering, Vol. 2(4) (1994)

[11] Nogales, F.J. et al, "Forecasting Next-Day Electricity Prices by Time Series Models", IEEE Transactions on Power Systems, Vol. 17, No. 2, May 2002, pp. 342-348.

[12] D.J. Sobajic and Y.H. Pao, "Artificial neural-net based dynamic security assessment for electric power systems," IEEE Transactions on Power Systems, vol. 4, pp. 220-228, 1989.

[13] M. El-Sharkawi and D. Neibur, "Artificial Neural Networks with applications to power systems,” IEEE-PES Special Publication 96TP-112-0, 1996.

[14] L. Wehenkel, Automatic Learning Techniques in Power Systems. Norwell, MA: Kluwer, 1998.

[15] N. Hatziargyriou, S. Papathanassiou, and M. Papadopoulos, "Decision trees for fast security assessment of autonomous power systems with large penetration from renewables," IEEE Trans. on Energy Conversion, vol. 10, June 1995.

[16] E.S. Karapidakis, N.D. Hatziargyriou, "Online Preventive Dynamic Security of Isolated Power Systems Using Decision Trees", IEEE Trans. on Power Systems, Vol 17, No 2, May 2002. 
[17] S. Papathanassiou, N. Hatziargyriou, K. Strunz, ”A Benchmark LV Microgrid for Steady State and Transient Analysis", Cigre Symposium "Power Systems with Dispersed Generation”, Athens, 17-20 April 2005

[18] ApX Historical Data, available on-line: http://www.apx.nl

\section{BIOGRAPHIES}

Nikos D. Hatziargyriou was born in Athens, Greece. He received the Diploma in Electrical and Mechanical Engineering from NTUA and MSc and PhD degrees from UMIST, Manchester, UK. He is professor at the Power Division of the Electrical and Computer Engineering Department of NTUA. His research interests include Dispersed and Renewable Generation, Dynamic Security Assessment, and application of Artificial Intelligence Techniques to power systems. He is senior IEEE member, member of CIGRE SCC6 and the Technical Chamber of Greece.

Aris L. Dimeas: was born in Athens, Greece in 1977. He received the diploma in Electrical and Computer Engineering from NTUA. He is currently a Ph.D. student at Electrical and Computers Engineering Department of NTUA. His research interests include dispersed generation and computer applications in liberalized energy markets. He is member of the Technical Chamber of Greece.

Antonis G. Tsikalakis was born in Athens, Greece in 1979. He received his diploma in Electrical and Computer Engineering from NTUA. He is currently a Ph.D. student at Electrical and Computers Engineering Department of NTUA. His research interests include optimization of power system operation, Dispersed Generation and energy storage. Mr. Tsikalakis is a student member of IEEE and member of the Technical Chamber of Greece

J. A. Peças Lopes is an Associate Professor with Aggregation in the Dept of EE of the Faculty of Engineering of University of Porto. He obtained an Electrical Engineering degree (5 years course) in 1981 from University of Porto and a PhD. degree also in Electrical Engineering from the same University in 1988. In 1996 he got an Aggregation degree. In 1989 he joined the staff of INESC as a senior researcher and he is presently Adjoint Coordinator of the Power Systems Unit of INESC Porto and a senior member of the IEEE.

G. N. Kariniotakis was born in Athens, Greece. He received his production and management engineering and MSc degrees from the Technical University of Crete, Greece and his PhD degree from Ecole des Mines de Paris in 1996. He is currently with the Center of Energy Studies of Ecole des Mines de Paris as a scientific projectmanager. He is a member of IEEE. His research interests include among others renewable energies, distributed generation and artificial intelligence.

José Oyarzabal was born in Bilbao,Spain, on 1967. He received the M.Sc.degree in Industrial Electrical Engineering,from the University of the Basque Country,Bilbao, in 1992. Since 1994 he has beenworking in Labein, involved in several applications related to power transmission and distribution control systems as well as protective relays. His special field of interest includes the application of information and communication technology standards to energy management systems. He is a member of the association of industrial engineers of Bilbao 